\title{
Evaluation of three spectroscopic techniques in determination of adulteration of cold pressed pomegranate seed oils
}

\author{
Oguz Uncu $^{\mathrm{a}}$, Alicja Napiórkowska ${ }^{\mathrm{b}}$, Tomasz K. Szajna ${ }^{\mathrm{b}}$, Banu Ozen ${ }^{\mathrm{a}, *}$ \\ ${ }^{a}$ Izmir Institute of Technology, Department of Food Engineering, Urla-Izmir, Turkey \\ ${ }^{\mathrm{b}}$ Warsaw University of Life Sciences, Institute of Food Sciences, Department of Food Technology, Warsaw, Poland
}

\section{A R T I C L E I N F O}

\section{Keywords:}

Pomegranate seed oil

Adulteration

Infrared spectroscopy

Fluorescence spectroscopy

UV-visible spectroscopy

\begin{abstract}
A B S T R A C T
It was aimed to compare three spectroscopic methods in determination of adulteration of cold pressed pomegranate seed oils (PSOs) with sunflower oil in this research. UV-visible, mid-infrared and fluorescence spectra of pure and adulterated pomegranate oils (1-50\%, v/v) were collected and data were analyzed with multivariate statistical analysis techniques. According to orthogonal partial least square discriminant analysis, best differentiation between pure and mixed samples was obtained with mid-infrared spectroscopy having 100\% success rate. Fluorescence and UV-visible spectroscopy also provided good discrimination between samples with 96 and $88 \%$ successful classification rates, respectively. As a result of partial least square regression analysis, detection limits for mid-infrared, UV-visible and fluorescence spectroscopies are determined as > 1, 5 and $10 \%$ in order. Since all spectroscopic methods provided detection of mixtures of cold pressed PSOs with sunflower oil at low concentrations they could serve as easy to use and rapid techniques in control laboratories.
\end{abstract}

\section{Introduction}

Pomegranate fruit is cultivated in global scale with the highest production in Mediterranean areas [1]. As a by-product of pomegranate fruit, pomegranate oil is produced from the seeds. This precious oil is considered as a good source of certain pharmaceutical and nutraceutical compounds [2]. There are various reports about the oil content of pomegranate seeds and literature indicates values between 4.4 and $24.1 \%[3,4]$. Pomegranate seed oil (PSO) is quite valuable owing to its unique fatty acid composition, which is very rich in terms polyunsaturated fatty acids, particularly punicic acid (18:3 cis 9 , trans 11, cis 13) as an isomer of conjugated linolenic acid [5]. Punicic acid composition of PSO varies approximately between 55 and 85\% [6,7]. This oil is also a very good source of various bioactive compounds such as tocopherols, phytosterols and squalene $[3,8,9]$. Many studies about the health effects of PSO indicate that this oil has various health benefits including antidiabetic, antiobesity, antiproliferative and anticarcinogenic effects originating mainly from pucinic acid along with other health promoting compounds [6]. These findings indicate that seed oils, especially PSO, could be used as functional ingredients not only in the food industry, but also in pharmaceutical and cosmetic fields [7].

As an economically valuable product, PSO has been subjected to mixing with cheaper and/or lower quality oils. Rapid, minimum waste generating with minimum sample preparation characteristics are desirable properties of spectroscopic methods for authentication studies of food and pharmaceutical products. There are several examples of the use of various spectroscopic methods such as infrared, nuclear magnetic resonance (NMR), Raman and fluorescence techniques in the adulteration detection of various types of high economic value oils [10-12]. Analysis of the spectral data with multivariate statistical techniques generally provide successful results with the condition that the data set has as many samples as possible covering the entire space of possible sample compositions. Although PSO is a very good target of adulteration due to its high price in the market, there is no study in the literature regarding the authentication of this product by any spectroscopic methods. The studies related with PSO are limited to determination of physicochemical properties $[2,7]$ as well as health benefits of this oil [6] without any authenticity concern.

It was aimed to compare the performances of three different spectroscopic methods (UV-visible, mid-infrared and fluorescence) for detection of mixtures of cold pressed PSO and sunflower oil by the evaluation of spectral data with multivariate statistical analysis techniques in this study.

\footnotetext{
* Corresponding author.

E-mail address: banuozen@iyte.edu.tr (B. Ozen).
} 


\section{Materials and methods}

\subsection{Oil samples and mixtures}

Fifteen cold pressed PSOs were obtained from trusted producers. As an adulterant, sunflower oil, was obtained from the local market. Sunflower oil was chosen as an adulterant due to its ease of availability in most parts of the world, its low price and ease of mixing with pomegranate oil without introducing any noticeable flavor. Seven of the randomly chosen pure PSO were blended with sunflower oil at 1, 5, 10, $15,20,30,40$ and $50 \%(\mathrm{v} / \mathrm{v})$ ratio; as a result, a total of 56 adulterated samples were prepared. Eight pure PSOs not included in the mixture set were independently used in the statistical models to test the capability of each model.

\subsection{Free fatty acid content}

Percent free fatty acid was determined by titration with $\mathrm{KOH}$ and calculated in terms of pucinic acid for PSO and oleic acid for sunflower oil according to a method from European Official Methods of Analysis [13]. The analyses were repeated two times.

\subsection{Fatty acid profile}

Fatty acid profiles of PSO and sunflower oil were determined with gas chromatographic (GC) analyses. Prior to chromatographic analyses, an esterification procedure was performed, and samples were injected into a GC device (Agilent 6890, Agilent Technologies, Santa Clara, CA, USA) with an auto-sampler (Agilent 7863 \& FID) and a split/splitless (1:50) injector. HP-88 capillary column (Agilent, USA) with dimensions

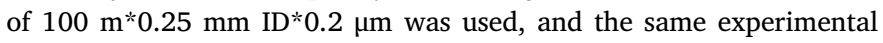
conditions were applied as in a previous study [14]. Results were calculated as percentages of corresponding fatty acid methyl esters with duplicate runs for each sample. The results comprise individual fatty acid profiles of PSOs as well as total saturated fatty acids (SFAs), total monounsaturated fatty acids (MUFAs), and total polyunsaturated fatty acids (PUFAs) contents.

\subsection{UV-visible spectroscopy}

UV-visible (UV-vis) range (200-800 nm) spectra of all samples were obtained with a UV-vis spectrophotometer (Shimadzu UV-2450 Spectrophotometer, Kyoto, Japan) with respect to an air background. Absorbance mode measurement parameters were $2.0 \mathrm{~nm}$ of sampling interval and slit width of $1 \mathrm{~nm}$, recorded by fast scanning in a semimicro type polystyrene cuvette with a $10 \mathrm{~mm}$ light path. Measurements were repeated twice for each oil.

\subsection{Mid-infrared spectroscopy}

After obtaining a background spectrum of air, mid-infrared (mid-IR) spectra of all samples were collected in the range of 4000-650 $\mathrm{cm}^{-1}$ with ZnSe-ATR attachment of Fourier transform infrared (FTIR) spectrometer (Spectrum 100 FTIR spectrometer, Perkin Elmer Inc., Waltham, MA, USA) having a DTGS detector. 64 scans of each spectrum with a resolution of $4 \mathrm{~cm}^{-1}$ and scan speed of $1 \mathrm{~cm} / \mathrm{s}$ were taken in two replicates. Between each run ATR crystal was cleaned with organic solvents and then dried with nitrogen.

\subsection{Fluorescence spectroscopy}

A fluorescence spectrometer (LS-55, PerkinElmer Inc., Waltham, MA, USA) having a pulsed xenon lamp was used in the collection of fluorescence spectra. Measurement parameters for the best resolution with optimal signal-to-noise ratio were slit width of 10 and $20 \mathrm{~nm}$ for excitation and emission, respectively and data interval for scan and integration time were $0.5 \mathrm{~nm}$ and $0.12 \mathrm{~s}$, respectively.

Emission spectra (300-800 nm) were collected for each excitation wavelengths $(320,330,340$, and $350 \mathrm{~nm}$ ) with quartz cell and measurements were repeated two times. The sample cell was cleaned with hexane under the flow of nitrogen between each run. Trial and error method was applied to select the optimal excitation wavelength to construct statistical models. Therefore, excitation at $340 \mathrm{~nm}$ was found as the most successful wavelength and all emission spectra excited at $340 \mathrm{~nm}$ were used in model building.

\subsection{Statistical analysis}

SIMCA 14.1 software (Umetrics, Umeå, Sweden) was used in both classification and prediction studies. Whole ranges of UV-vis $(200-800 \mathrm{~nm})$, FTIR (4000-650 $\left.\mathrm{cm}^{-1}\right)$ and fluorescence $(300-800 \mathrm{~nm})$ spectroscopic methods were analyzed individually and compared with each other in terms of their success in differentiation of pure PSO samples from adulterated ones and also in prediction of adulteration level of all these samples (pure and adulterated). Firstly, main preprocessing methods such as mean-centering and unit variance scaling as well as the most appropriate pre-treatment techniques as second derivative (SD), wavelet denoising techniques (WDTs) in combination with SD (WDTs:SD) and orthogonal signal correction (OSC) were applied in the development of the specific statistical models to remove any noise of the averaged data. SD of the spectroscopic data was based on moving quadratic sub-models of 15 data point long with a distance of 1 excluding the edge effects. As a WDTs, Daubechies-10 was selected at the $99.5 \%$ confidence interval. Mean-centering and unit variance scaling were applied to all spectroscopic data.

All the pre-treated data set of each spectroscopic technique used in both classification and prediction purposes was randomly divided into calibration and validation sets comprising $2 / 3$ and $1 / 3$ number of the data set, respectively. The number of latent variables (LVs) used in construction of calibration data sets was optimized by internal validation (cross validation) in the form of leave-one-out cross validation (LOO-CV) in order to avoid over and/or under fitting of the model [15].

For the classification purposes, orthogonal partial least square-discriminant analysis (OPLS-DA) was used as a chemometric tool to reveal the separation of adulterated and pure PSO samples by using pretreated data. In the OPLS-DA, a correlation model was built from two main matrix block consisting a defined $\mathrm{X}$ matrix (spectral data) and an artificially constructed dummy Y matrix (variable vector defined as class 1 for pure (non-adulterated) and as class 2 for adulterated samples) [16]. Outcome of the OPLS-DA analysis was interpreted with classification tables revealing correct classification rate (\%CC). \%CC was calculated manually if any examined sample from a known oil class (as adulterated or non-adulterated) have a prediction value between 0.5 and 1.5; otherwise, it was considered as a misclassification [17]. Other performance parameters were calculated by the SIMCA software automatically such as the LVs, coefficient of determination for calibration $\left(\mathrm{R}_{\text {cal. }}^{2}\right)$, coefficient of determination for cross validation $\left(\mathrm{R}_{\mathrm{cv} .}^{2}\right)$ of each classification model.

Prediction studies of varying level of adulteration between 0 and $50 \%$ in volume base were conducted by partial least squares (PLS) regression. PLS regression stands for correlating spectroscopic data of all the studied samples including adulterated and non-adulterated (X matrix) with the level of adulterated (1-50\%) and non-adulterated (0\%) PSO samples (Y matrix) [18]. Quantification ability of PLS models was inspected with various statistical parameters such as $R_{\text {cal., }}^{2} R_{\text {cv. }}^{2}, R_{\text {pred. }}^{2}$ for external validation, root mean square error of calibration (RMSEC), root mean square error of cross-validation (RMSECV), root mean square error of prediction (RMSEP), residual predictive deviation (RPD), and the slope. Robust quantification models could only be obtained with high regression coefficient $>0.90$ indicating excellent predictions [19]. A slope value in the range of 0.9-1.1 and RPD value higher than 3.0 show a very reliable prediction model [19]. On the other hand, 
Table 1

Chemical parameters of pure pomegranate seed oil samples.

\begin{tabular}{|c|c|c|c|c|c|c|c|c|c|c|c|c|c|c|c|}
\hline Sample & FFA $^{1}$ & $\mathrm{C} 16: 0^{2}$ & C18: $0^{3}$ & $C 18: 1 n 9 c^{4}$ & $C 18: 2 n 6 c^{5}$ & C20: $0^{6}$ & $\mathrm{C} 18.3 \mathrm{n} 3^{7}$ & $\mathrm{C} 20: 3 \mathrm{n} 6^{8}$ & C20:4n $6^{9}$ & $\mathrm{C} 18: 3^{10}$ & $\mathrm{C} 18: 3^{11}$ & $\mathrm{C} 18: 3^{12}$ & $\mathrm{SFAs}^{13}$ & MUFAs $^{14}$ & PUFAs $^{15}$ \\
\hline PSO(A) & 3.30 & 2.74 & 2.07 & 4.50 & 4.67 & 0.46 & 0.72 & 0.10 & 0.32 & 69.45 & 10.42 & 4.07 & 5.33 & 4.50 & 89.76 \\
\hline $\mathrm{PSO}(\mathrm{B})$ & 0.70 & 2.52 & 1.88 & 4.06 & 4.14 & 0.42 & 0.68 & 0.08 & 0.31 & 79.44 & 5.60 & 0.54 & 4.87 & 4.06 & 90.80 \\
\hline $\mathrm{PSO}(\mathrm{C})$ & 0.58 & 2.76 & 2.19 & 5.26 & 5.40 & 0.41 & 0.59 & 0.09 & 0.27 & 81.12 & 1.45 & 0.12 & 5.41 & 5.26 & 89.04 \\
\hline PSO(D) & 2.00 & 2.66 & 2.00 & 4.27 & 4.40 & 0.43 & 0.69 & 0.09 & 0.29 & 74.84 & 7.80 & 2.22 & 5.14 & 4.27 & 90.32 \\
\hline PSO(E) & 1.94 & 2.79 & 2.22 & 5.00 & 5.12 & 0.42 & 0.63 & 0.09 & 0.22 & 76.52 & 5.21 & 1.74 & 5.48 & 5.00 & 89.52 \\
\hline $\mathrm{PSO}(\mathrm{F})$ & 1.53 & 2.71 & 2.09 & 4.65 & 4.74 & 0.44 & 0.67 & 0.08 & 0.25 & 76.64 & 6.03 & 1.66 & 5.28 & 4.65 & 90.07 \\
\hline PSO(G) & 3.43 & 2.75 & 2.06 & 5.31 & 6.96 & 0.46 & 0.63 & 0.11 & 0.36 & 72.34 & 7.36 & 1.63 & 5.32 & 5.31 & 89.38 \\
\hline $\mathrm{PSO}(\mathrm{H})$ & 0.64 & 2.64 & 2.03 & 4.66 & 4.77 & 0.42 & 0.64 & 0.09 & 0.29 & 80.28 & 3.53 & 0.33 & 5.14 & 4.66 & 89.92 \\
\hline PSO(I) & 3.37 & 2.75 & 2.06 & 4.90 & 5.82 & 0.46 & 0.67 & 0.10 & 0.34 & 70.89 & 8.89 & 2.85 & 5.32 & 4.90 & 89.57 \\
\hline $\mathrm{PSO}(\mathrm{J})$ & 2.07 & 2.64 & 1.97 & 4.68 & 5.55 & 0.44 & 0.66 & 0.10 & 0.34 & 75.89 & 6.48 & 1.08 & 5.09 & 4.68 & 90.09 \\
\hline PSO(K) & 2.01 & 2.76 & 2.12 & 5.28 & 6.18 & 0.43 & 0.61 & 0.10 & 0.31 & 76.73 & 4.41 & 0.88 & 5.36 & 5.28 & 89.21 \\
\hline PSO(L) & 2.72 & 2.71 & 2.03 & 4.79 & 5.68 & 0.44 & 0.66 & 0.10 & 0.32 & 73.59 & 7.58 & 1.93 & 5.23 & 4.79 & 89.85 \\
\hline PSO(M) & 2.69 & 2.77 & 2.14 & 5.15 & 6.04 & 0.44 & 0.63 & 0.10 & 0.29 & 74.43 & 6.28 & 1.69 & 5.40 & 5.15 & 89.45 \\
\hline $\mathrm{PSO}(\mathrm{N})$ & 2.48 & 2.73 & 2.07 & 4.98 & 5.85 & 0.45 & 0.65 & 0.09 & 0.30 & 74.49 & 6.69 & 1.65 & 5.30 & 4.98 & 89.72 \\
\hline $\mathrm{PSO}(\mathrm{O})$ & 2.04 & 2.70 & 2.05 & 4.98 & 5.86 & 0.44 & 0.63 & 0.10 & 0.33 & 76.31 & 5.44 & 0.98 & 5.23 & 4.98 & 89.65 \\
\hline Average & 2.10 & 2.71 & 2.07 & 4.83 & 5.41 & 0.44 & 0.65 & 0.09 & 0.30 & 75.53 & 6.21 & 1.56 & 5.26 & 4.83 & 89.76 \\
\hline SD & 0.91 & 0.07 & 0.08 & 0.36 & 0.74 & 0.02 & 0.03 & 0.01 & 0.04 & 3.15 & 2.10 & 0.98 & 0.15 & 0.36 & 0.43 \\
\hline Min & 0.58 & 2.52 & 1.88 & 4.06 & 4.14 & 0.41 & 0.59 & 0.08 & 0.22 & 69.45 & 1.45 & 0.12 & 4.87 & 4.06 & 89.04 \\
\hline Max & 3.43 & 2.79 & 2.22 & 5.31 & 6.96 & 0.46 & 0.72 & 0.11 & 0.36 & 81.12 & 10.42 & 4.07 & 5.48 & 5.31 & 90.80 \\
\hline
\end{tabular}

${ }^{1}$ Free fatty acid, ${ }^{2}$ palmitic acid, ${ }^{3}$ stearic acid, ${ }^{4}$ oleic acid, ${ }^{5}$ linoleic acid, ${ }^{6}$ arachidic acid, ${ }^{7}$ linolenic acid, ${ }^{8}$ cis $8,11,14$-eicosatrienoic acid, ${ }^{9}$ arachidonic acid, ${ }^{10}$ punicic acid, ${ }^{11} \alpha$-eleostearic acid, ${ }^{12}$ catalpic acid, ${ }^{13}$ saturated fatty acids, ${ }^{14}$ monounsaturated fatty acids, ${ }^{15}$ polyunsaturated fatty acids.

Table 2

Statistical parameters of OPLS-DA calibration and validation models of pure and adulterated pomegranate seed oil samples.

\begin{tabular}{|c|c|c|c|c|c|c|c|c|c|c|}
\hline \multirow[t]{3}{*}{ Model } & \multirow[t]{3}{*}{ Number of samples } & \multicolumn{3}{|c|}{ UV-vis } & \multicolumn{3}{|l|}{ FTIR } & \multicolumn{3}{|c|}{ Fluorescence } \\
\hline & & \multicolumn{3}{|c|}{$\begin{array}{l}\text { Pre-treatment: SD, LVs: } 1+5 \\
\mathrm{R}_{\text {cal. }}^{2}: 0.87, \mathrm{R}_{\mathrm{cv} .}^{2}: 0.19\end{array}$} & \multicolumn{3}{|c|}{$\begin{array}{l}\text { Pre-treatment: SD, LVs: } 1+2 \\
\mathrm{R}_{\text {cal. }}^{2}: 0.96, \mathrm{R}_{\mathrm{cv} .}^{2}: 0.51\end{array}$} & \multicolumn{3}{|c|}{$\begin{array}{l}\text { Pre-treatment: WDTs:SD, LVs: } 1+5 \\
\mathrm{R}_{\text {cal. }}^{2}: 0.96, \mathrm{R}_{\mathrm{cv} .}^{2}: 0.58\end{array}$} \\
\hline & & Pure & Adulterated & $\% \mathrm{CC}$ & Pure & Adulterated & $\% \mathrm{CC}$ & Pure & Adulterated & $\% \mathrm{CC}$ \\
\hline \multicolumn{11}{|l|}{ Calibration } \\
\hline Pure & 10 & 10 & 0 & 100 & 10 & 0 & 100 & 10 & 0 & 100 \\
\hline Adulterated & 37 & 0 & 37 & 100 & 0 & 37 & 100 & 0 & 37 & 100 \\
\hline Total & 47 & 10 & 37 & 100 & 10 & 37 & 100 & 10 & 37 & 100 \\
\hline \multicolumn{11}{|l|}{ Validation } \\
\hline Pure & 5 & 4 & 1 & 80 & 5 & 0 & 100 & 4 & 1 & 80 \\
\hline Adulterated & 19 & 2 & 17 & 89 & 0 & 19 & 100 & 0 & 19 & 100 \\
\hline Total & 24 & 6 & 18 & 88 & 5 & 19 & 100 & 4 & 20 & 96 \\
\hline
\end{tabular}

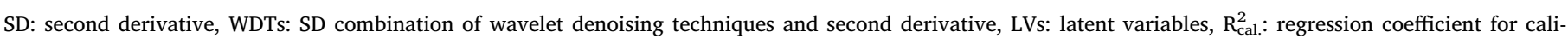
bration, $\mathrm{R}_{\mathrm{cv}}^{2}$ : regression coefficient for cross validation, \%CC: average correct classification rate.

there are no exact ranges for the error values because they are dependent on the range and magnitude of the reference value to be predicted. Instead, comparably low and close error values of calibration (RMSEC), cross validation (RMSECV) and external validation (RMSEP) are expected for a good model [14]. All these parameters except RPD were included in the statistical software. RPD is a comprehensive value to reveal the prediction ability of the corresponding model in terms of the ratio of standard deviation of predicted values to RMSEP values [15]. The RPD values were calculated manually as shown in the literature [20].

\section{Results and discussion}

Free fatty acid values and fatty acid profiles of pomegranate oils were determined to obtain information about the characteristics of the oils analyzed and the ranges of these parameters for pure PSOs are shown in Table 1 . Free fatty acid values of the pure samples range between 0.58 and $3.43 \%$. A study in the literature reported values between 1.80 and $4.38 \%$ for free fatty acid content of cold pressed PSOs from two different countries [1]. Punicic acid is the most common fatty acid of the oil samples followed by $\alpha$-eleostearic and linolenic acid and pucinic acid content varies between 69.45 and $81.12 \%$ for the pure oils. Ranges for total MUFAs and PUFAs are 4.06-5.31\% and 89.04-90.80\%, respectively (Table 1 ). These ranges are mostly in agreement with the values in the literature $[1,3,4,7,9]$. Quantitative differences could be attributed to growing location [21] as well as inter-varietal differences of PSO samples [22]. Some chemical characteristics of sunflower oil used as an adulterant were also measured. Free fatty acid value of sunflower oil was determined as $0.08 \%$ and major fatty acids of the sunflower oil were found as palmitic (6.36\%), stearic (3.43\%), oleic (30.93\%) and linoleic acids (57.92\%), and total SFA, MUFA, and PUFA contents were $10.81 \%, 31.11 \%$ and $58.08 \%$, respectively. Oleic and linoleic acid contents of sunflower oil were much higher than PSOs whereas PUFA content of PSOs was higher compared to sunflower oil. In addition, the characteristic fatty acid compounds of PSOs such as pucinic acid was not found in the sunflower oil. These significant differences in fatty acid composition could also be used as markers in adulteration detection and they are also highly correlated with the spectral differences of the studied oils as will be discussed in the next parts.

Spectra of 71 samples (15 pure and 56 adulterated) were collected using three spectroscopic methods, UV-vis, mid-IR and fluorescence. These spectral data were analyzed with multivariate statistical analysis techniques, OPLS-DA and PLS for classification and prediction purposes, respectively. Statistical parameters of constructed models for each spectroscopic approach are listed in Table 2 for classification and Table 3 for prediction. Different pre-treatments are applied to spectral data and only the results of the best transformations are provided. 
Table 3

Statistical parameters of PLS regression models for prediction of pomegranate seed oil adulteration by spectroscopic methods.

\begin{tabular}{|c|c|c|c|c|c|c|c|c|c|c|}
\hline Method & Pre-treatment & LVs & $\mathrm{R}^{2}$ cal. & $\mathrm{R}_{\mathrm{cv}}^{2}$ & $\mathrm{R}_{\text {pred. }}^{2}$ & RMSEC & RMSECV & RMSEP & RPD & Slope \\
\hline UV-vis & SD & 5 & 0.98 & 0.93 & 0.98 & 2.29 & 4.94 & 2.78 & 5.94 & 0.98 \\
\hline FTIR & SD & 3 & 0.99 & 0.99 & 0.99 & 0.57 & 1.51 & 1.42 & 12.48 & 0.99 \\
\hline Fluorescence & OSC & 4 & 0.96 & 0.94 & 0.96 & 3.19 & 4.48 & 3.59 & 4.77 & 0.96 \\
\hline
\end{tabular}

SD: second derivative, OSC: orthogonal signal correction, LVs: latent variables, $\mathrm{R}_{\text {cal. }}^{2}$ : regression coefficient for calibration, $\mathrm{R}_{\text {cv. }}^{2}$ : regression coefficient for cross validation, $\mathrm{R}_{\text {pred.: }}^{2}$ regression coefficient for prediction (external val.), RMSEC: root mean square error of calibration, RMSECV: root mean square error of crossvalidation, RMSEP: root mean square error of prediction, RPD: residual predictive deviation.

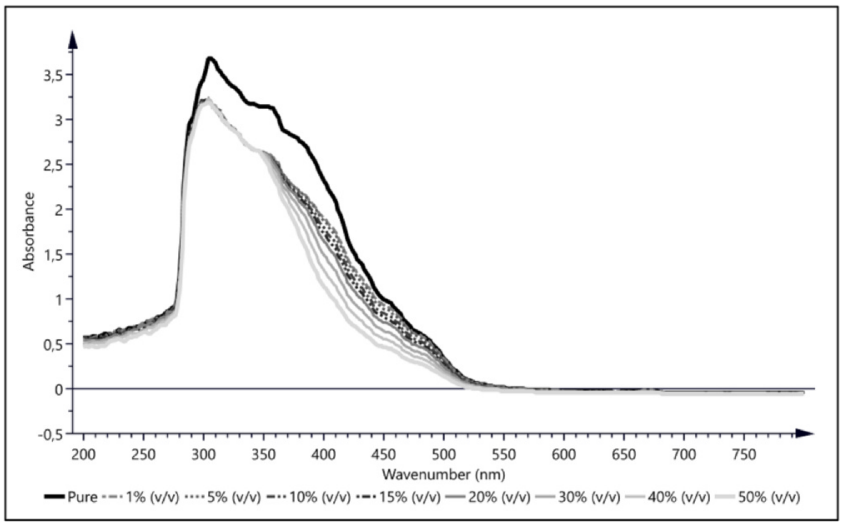

(a)

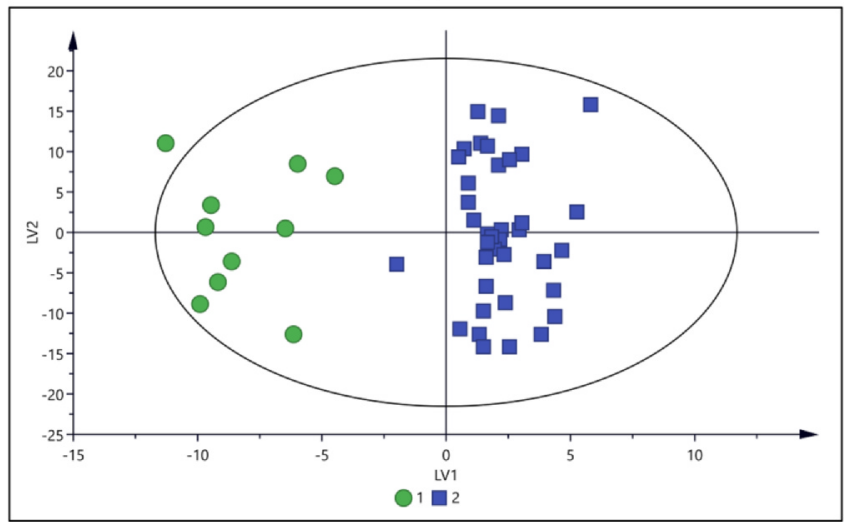

(b)

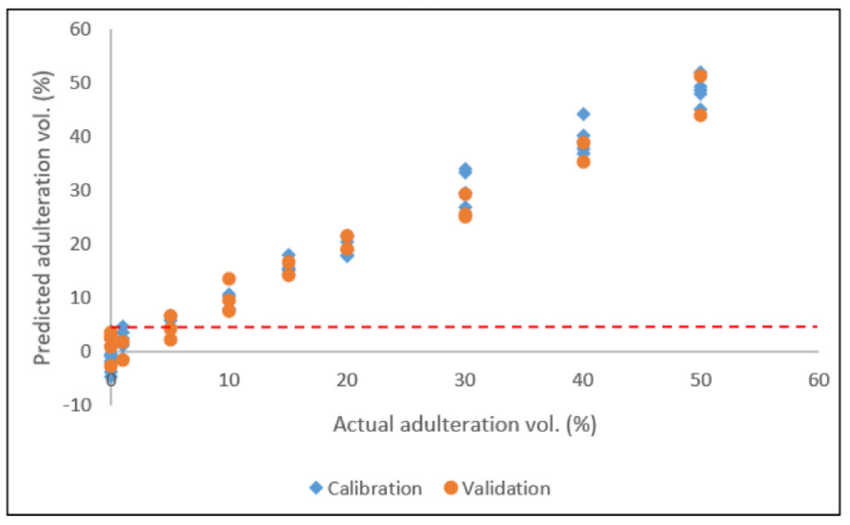

(c)

Fig. 1. a) UV-visible spectra of pure and adulterated pomegranate seed oils; b) OPLS-DA score plot constructed with UV-visible spectral data $(\bigcirc 1$ : pure and 2: adulterated pomegranate seed oil samples); c) PLS regression plot of predicted vs actual adulteration concentration for UV-visible spectroscopy.
Every model has a calibration set containing 47 samples and a validation set of 24 oils.

\subsection{UV-visible spectroscopy}

Averaged raw UV-visible spectra of pure and adulterated samples are provided in Fig. 1a. Major changes in the absorbance values of adulterated samples with respect to pure oils are in $300-540 \mathrm{~nm}$ region (Fig. 1a). While peaks in $300-400 \mathrm{~nm}$ region are associated with polyphenolic compounds, absorptions at $430-460 \mathrm{~nm}$ correspond to carotenoids [23]. As can be seen from the Fig. 1a, there is a clear decreasing trend in absorbance values with increasing adulteration concentration. Therefore, any differentiation with respect to adulteration could be associated with the absorbance values in these regions for UV-visible spectroscopy. There are various reports in the literature showing the high phenolic and carotenoid contents of PSO $[3,8,9]$. Moreover, PSOs have been proposed as a more effective alternative to synthetic antioxidants to protect plant-based oils against oxidation [24].

Pre-treatment of UV-visible spectra with SD provided the best results for classification model and OPLS-DA model generated after this transformation have 1 predictive and 5 orthogonal components (Table 2). According to the score plot of this model (Fig. 1b), pure and adulterated samples are clearly separated from each other with respect to LV1 as being in the left and right side of the score plot, respectively except one sample having $1 \%$ adulterant. Correct classification table (Table 2) indicates that $100 \%$ of all samples in calibration set are correctly classified within their group while $88 \%$ success rate (1 pure oil is misclassified as adulterated out of 5 samples and 2 adulterated samples are wrongly classified as pure out of 19 samples) is obtained for external validation. In multivariate statistical analysis, variable importance in projection (VIP) scores $>1$ indicate the significance of variables under investigation. VIP values of OPLS-DA of UV-vis spectral data confirmed that $300-400 \mathrm{~nm}$ (polyphenols) and $400-500 \mathrm{~nm}$ (carotenoids) are the most significant regions which caused separation of the samples.

UV-vis spectral data is also evaluated with PLS regression to construct a correlation between actual and predicted percentages of mixing in order to see quantification ability of the corresponding model. Statistical parameters of PLS regression model are provided in Table 3 and regression plot is shown in Fig. 1c. The best pre-treatment for PLS model is SD and this model is constructed with $5 \mathrm{LVs}$ having the lowest $\mathrm{R}^{2}$ of 0.93 . RPD value of 5.94 is an indication of a robust model along with slope (0.98) close to 1 . In addition, RMSE values (2.29-4.94\%) are low enough and close to each other (Table 3). Examination of PLS regression plot indicated that detection of adulteration $>5 \%$ could be possible with the use of UV-vis spectroscopy (Fig. 1c). Although it is a very economical and easy to use technique, UV-vis spectroscopy is not as common as other spectroscopic methods in detection of adulteration of cold pressed oils and has never been used for authentication of PSO before. Among the few studies in this area, UV-vis spectra also provided good separation between fresh and fresh-old olive oil mixtures [25]. 


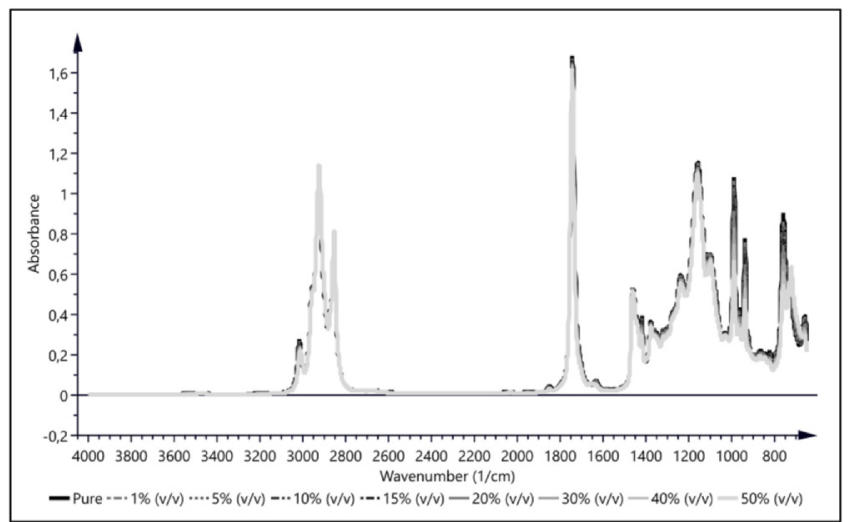

(a)

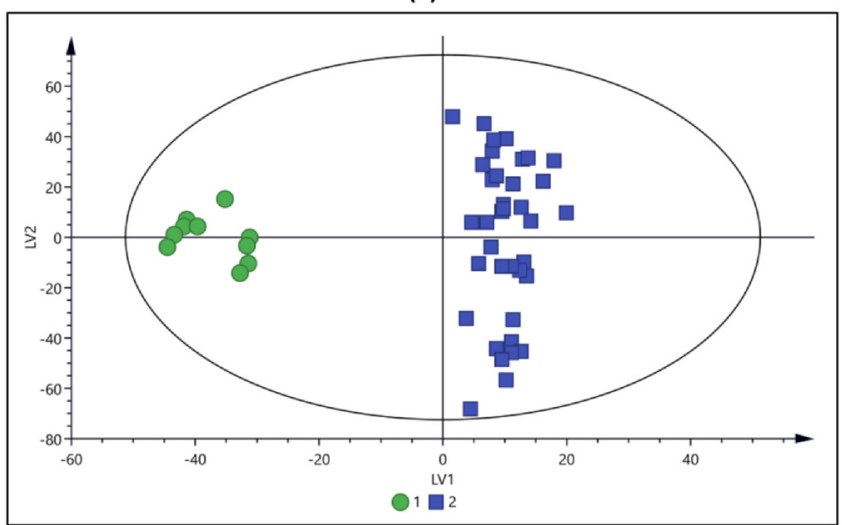

(b)

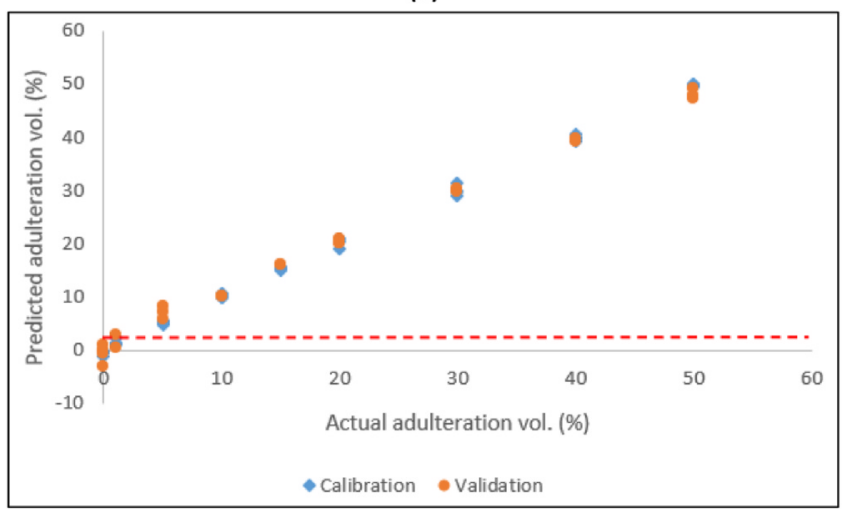

(c)

Fig. 2. a) Mid-IR spectra of pure and adulterated pomegranate seed oils; b) OPLS-DA score plot constructed with mid-IR spectral data (O1: pure and $\square$ : adulterated pomegranate seed oil samples); c) PLS regression plot of predicted vs actual adulteration concentration for mid-IR spectroscopy.

\subsection{Mid-IR spectroscopy}

Differences in the peaks of pure and adulterated samples are at around $2924 \mathrm{~cm}^{-1}, 2852 \mathrm{~cm}^{-1}, 1723 \mathrm{~cm}^{-1}$, as well as fingerprint region (1464-983 $\mathrm{cm}^{-1}$ ) and $723 \mathrm{~cm}^{-1}$ (Fig. 2a) of mid-IR wavenumber ranges. Pure samples showed higher absorption at some of these wavelengths because of different chemical characteristics of PSO samples compared to adulterant, sunflower oil. First, due to refining process sunflower oil does not contain considerable amounts of free fatty acids whereas cold pressed PSO samples do. At $1723 \mathrm{~cm}^{-1}$ an observable peak exists based on stretching of $\mathrm{C}=\mathrm{O}$ groups correlated with free fatty acids [17] and this peak shows decreasing trend with increasing adulterant level. In addition, absorbances at the fingerprint region (1464-983 $\mathrm{cm}^{-1}$ ) and around $722 \mathrm{~cm}^{-1}$ of the PSO samples were found higher compared to adulterated samples due to fact that these particular wavenumbers are associated with polyphenols and tocopherols [26]. On the other hand, there is a positive correlation between increasing adulterant level and the absorbances at around 2924 and $2852 \mathrm{~cm}^{-1}$ based on symmetrical and asymmetrical stretching vibrations of $\mathrm{CH}_{2}$, respectively [25].

Mid-IR data are analyzed to differentiate pure and mixed samples with OPLS-DA (Table 2). Score plot of OPLS-DA model is shown in Fig. $2 \mathrm{~b}$ and this plot indicates a very good separation between pure and mixed oils with respect to the first $\mathrm{LV}$ explaining $37 \%$ of the total variance. Correct classification table also confirms this observation (Table 2). According to this table, all of the samples in both calibration and external validation sets are classified in their own group with $100 \%$ success rate. The most significant wavelengths in discrimination of pure and adulterated samples are explained with VIP values. The highest VIP values are obtained at fingerprint region as well as 2924 and $2852 \mathrm{~cm}^{-1}$ along with the major peak at around $1743 \mathrm{~cm}^{-1}$.

PLS regression model constructed using SD of mid-IR spectral data with 3 LVs have $R^{2}$ values of 0.99 and low and close RMSE values (Table 3). Slope of the regression line, 0.99 , is close to 1 and RPD value (12.48) is quite high confirming excellent prediction ability (Table 3). Therefore, it could be concluded that a robust and accurate model is obtained. As PLS regression plot indicates, detection of mixtures of PSO with sunflower oil is possible above $1 \%$ level (Fig. 2c), which is a quite satisfactory threshold value for an adulteration study. As in our study, mid-IR spectroscopy has been a quite successful technique regarding the determination of adulteration of different types of cold pressed oils such as sesame, black cumin and avocado oils with low detection levels [27-29]. To the best of our knowledge, there is not any comparable PSO adulteration and/or characterization study using FTIR analysis in the literature.

\subsection{Fluorescence spectroscopy}

Fluorescence spectroscopic profiles of pure and adulterated oils are presented in Fig. 3a. $350-700 \mathrm{~nm}$ range is the region where major differences between mixed and pure samples are observed visually. $250-400 \mathrm{~nm}$ region are mostly associated with $\alpha$-tocopherol and phenolic compounds while $400-600 \mathrm{~nm}$ part of the spectra is attributed to carotenoids along with fatty acid oxidation products [30,31]. The fluorescent intensity in the $400-600 \mathrm{~nm}$ range correlates positively with the increasing mixture levels due to more oxidative fluorophore compounds of these samples.

OPLS-DA of fluorescence spectral profile resulted a model with 1 predictive and 5 orthogonal components. OPLS-DA score plot indicates a good differentiation between pure and adulterated oils (Fig. 3b). Only one of the samples having $1 \%$ sunflower oil is on the line of the first LV. Other samples in two groups are well separated with respect to the first LV explaining $42 \%$ of the total variance. Model placed $100 \%$ of the samples in the calibration set to the right class while $96 \%$ success rate was obtained in the external validation set due to the misplacement of 1 pure sample to the adulterated class (Table 2). VIP values indicated that $350-600 \mathrm{~nm}$ range and minor peak around $670 \mathrm{~nm}$ are responsible for differentiation.

Four component PLS regression model of fluorescence spectral data pre-processed with OSC have $\mathrm{R}^{2}$ values $\geq 0.94$ (Table 3 ). RMSE values are in $3.19-4.48 \%$ range which are higher compared to mid-IR PLS model but closer to UV-vis model; however, these values are still in acceptable range. PLS model for fluorescence data have the lowest RPD value (4.77) compared to UV-vis (5.94) and mid-IR (12.48) models (Table 3); nevertheless, this RPD is high enough for a robust model. Evaluation of PLS regression plot (Fig. 3c) reveals that determination of addition of sunflower oil to PSO at higher than $10 \%$ level could be possible with this analysis. Fluorescence spectroscopy has also various applications in detection of mixtures of cold pressed oils with cheaper oils $[27,32]$. As opposed to this study, synchronous fluorescence spectroscopy provided better results compared to mid-IR spectroscopy in 


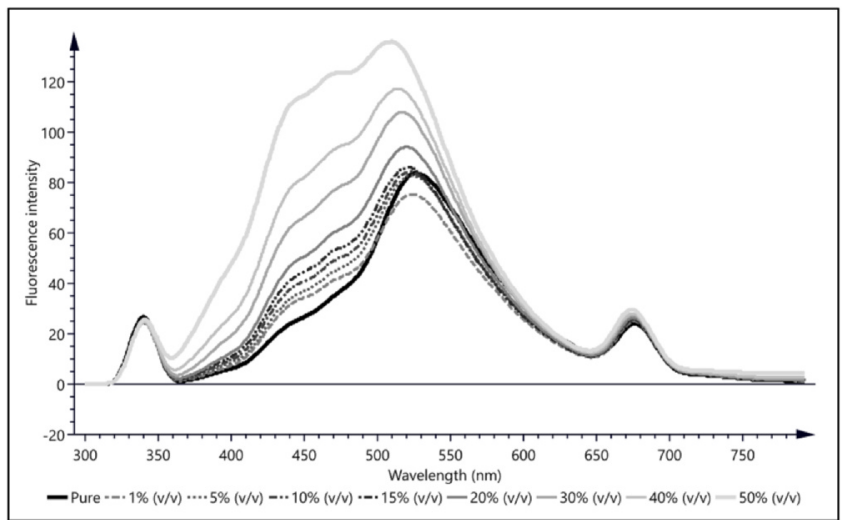

(a)

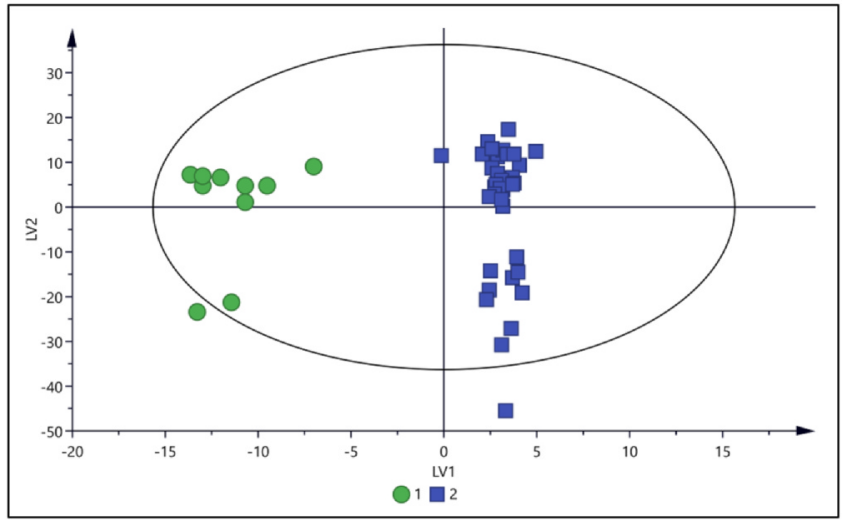

(b)

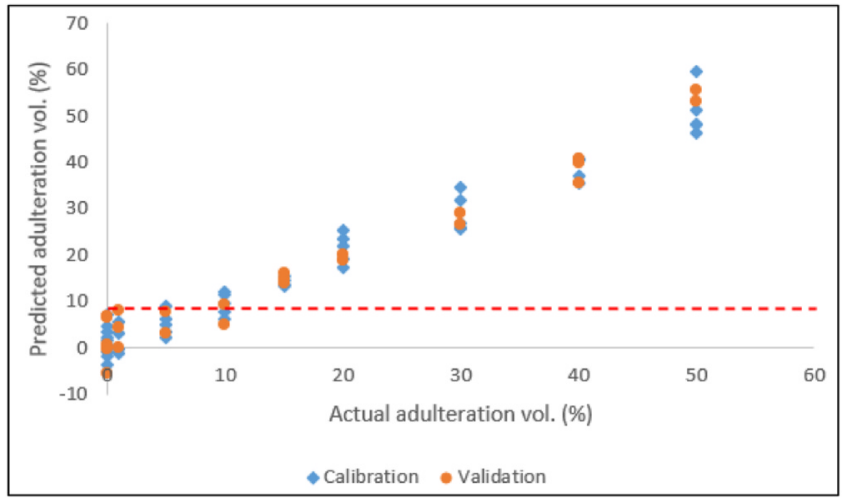

(c)

Fig. 3. a) Fluorescence spectra of pure and adulterated pomegranate seed oils; b) OPLS-DA score plot constructed with fluorescence spectral data (O1: pure and 2: adulterated pomegranate seed oil samples); c) PLS regression plot of predicted vs actual adulteration concentration for fluorescence spectroscopy.

detection of adulteration of black cumin oil with soybean oil with a detection limit of 5\% [27]. However, fluorescence spectroscopy has not been used in any study related with the authentication of PSO before.

\section{Conclusions}

Mid-IR spectroscopy provided the best results regarding the determination of mixing of cold pressed PSO with sunflower oil. Mid-IR spectroscopy presents a holistic approach which has contributions from all constituents of the samples. However, statistical models based on UV-vis spectroscopy depends mostly on pigment profiles of the samples. Although mid-IR spectroscopy has the lowest detection limit UV-vis and fluorescence spectroscopy techniques still allow determination of mixing at low levels. Therefore, all 3 methods could be used as an authentication tool for cold pressed PSOs.

\section{CRediT authorship contribution statement}

Oguz Uncu: Methodology, Formal analysis, Investigation, Writing original draft, Writing - review \& editing, Supervision. Alicja Napiórkowska: Investigation. Tomasz K. Szajna: Investigation. Banu Ozen: Conceptualization, Methodology, Formal analysis, Investigation, Writing - original draft, Writing - review \& editing, Resources, Supervision, Project administration, Funding acquisition.

\section{Declaration of Competing Interest}

The authors declare that they have no known competing financial interests or personal relationships that could have appeared to influence the work reported in this paper.

\section{Acknowledgements}

We would like to thank Chemical Engineering Department and Environmental Research Center of Izmir Institute of Technology for their help for fluorescence spectroscopy and GC analyses, respectively.

\section{References}

[1] A.M.M. Costa, L.O. Silva, A.G. Torres, Chemical composition of commercial coldpressed pomegranate (Punica granatum) seed oil from Turkey and Israel, and the use of bioactive compounds for samples' origin preliminary discrimination, J. Food Compos. Anal. 75 (2019) 8-16, https://doi.org/10.1016/j.jfca.2018.09.004.

[2] A. Khoddami, Y.B.C. Man, T.H. Roberts, Physico-chemical properties and fatty acid profile of seed oils from pomegranate (Punica granatum L.) extracted by cold pressing, Eur. J. Lipid Sci. Technol. 116 (2014) 553-562, https://doi.org/10.1002/ ejlt.201300416

[3] L. Fernandes, J.A. Pereira, I. Lopéz-Cortés, D.M. Salazar, E. Ramalhosa, S. Casal, Fatty acid, vitamin E and sterols composition of seed oils from nine different pomegranate (Punica granatum L.) cultivars grown in Spain, J. Food Compos. Anal. 39 (2015) 13-22, https://doi.org/10.1016/j.jfca.2014.11.006.

[4] M. Kýralan, M. Gölükcü, H. Tokgöz, Oil and conjugated linolenic acid contents of seeds from important pomegranate cultivars (Punica granatum L.) grown in Turkey, J. Am. Oil Chem. Soc. 86 (2009) 985-990, https://doi.org/10.1007/s11746-0091436-x.

[5] I.L.P. Melo, E.B.T. Carvalho, J. Mancini-Filho, Pomegranate seed oil (Punica granatum L.): a source of punicic acid (conjugated $\alpha$-linolenic acid), J. Hum. Nutr. Food Sci. 2 (2014) 1024.

[6] P. Aruna, D. Venkataramanamma, A.K. Singh, R.P. Singh, Health benefits of punicic acid: a review, Compr. Rev. Food Sci. Food Saf. 15 (2016) 16-27, https://doi.org/ 10.1111/1541-4337.12171.

[7] F. Siano, M.C. Straccia, M. Paolucci, G. Fasulo, F. Boscaino, M.G. Volpe, Physicochemical properties and fatty acid composition of pomegranate, cherry and pumpkin seed oils, J. Sci. Food Agric. 96 (2016) 1730-1735, https://doi.org/10. $1002 /$ jsfa.7279.

[8] A. Caligiani, F. Bonzanini, G. Palla, M. Cirlini, R. Bruni, Characterization of a potential nutraceutical ingredient: pomegranate (Punica granatum L.) seed oil unsaponifiable fraction, Plant Foods Hum. Nutr. 65 (2010) 277-283, https://doi.org/ 10.1007/s11130-010-0173-5.

[9] V. Verardo, P. Garcia-Salas, E. Baldi, A. Segura-Carretero, A. Fernandez-Gutierrez, M.F. Caboni, Pomegranate seeds as a source of nutraceutical oil naturally rich in bioactive lipids, Food Res. Int. 65 (2014) 445-452, https://doi.org/10.1016/j. foodres.2014.04.044.

[10] H. Chen, Z. Lin, C. Tan, Fast quantitative detection of sesame oil adulteration by near-infrared spectroscopy and chemometric models, Vib. Spectrosc. 99 (2018) 178-183, https://doi.org/10.1016/j.vibspec.2018.10.003.

[11] T. Shi, M. Zhu, Y. Chen, X. Yan, Q. Chen, X. Wu, J. Lin, M. Xie, ${ }^{1}$ H NMR combined with chemometrics for the rapid detection of adulteration in camellia oils, Food Chem. 242 (2018) 308-315, https://doi.org/10.1016/j.foodchem.2017.09.061.

[12] P. Vargas Jentzsch, F. Gualpa, L.A. Ramos, V. Ciobotă, Adulteration of clove essential oil: Detection using a handheld Raman spectrometer, Flavour, Fragrance J. 33 (2018) 184-190, https://doi.org/10.1002/ffj.3438.

[13] European Community, Commission Regulation (EEC) No 2568 / 91 of 11 July 1991 on the characteristics of olive oil and olive-residue oil and on the relevant methods of analysis, Off. J. Eur. Commun. L248 (1991) 1-83.

[14] O. Uncu, B. Ozen, Prediction of various chemical parameters of olive oils with Fourier transform infrared spectroscopy, LWT-Food Sci. Technol. 63 (2015) 978-984, https://doi.org/10.1016/j.lwt.2015.05.002.

[15] J. Riedl, S. Esslinger, C. Fauhl-Hassek, Review of validation and reporting of nontargeted fingerprinting approaches for food authentication, Anal. Chim. Acta 885 (2015) 17-32, https://doi.org/10.1016/j.aca.2015.06.003.

[16] I. Sen, F. Tokatli, Differentiation of wines with the use of combined data of UV-visible spectra and color characteristics, J. Food Compos. Anal. 45 (2016) 101-107, https://doi.org/10.1016/j.jfca.2015.09.018. 
[17] A. Hirri, M. Bassbasi, S. Platikanov, R. Tauler, A. Oussama, FTIR spectroscopy and PLS-DA classification and prediction of four commercial grade virgin olive oils from Morocco, Food Anal. Methods 9 (2016) 974-981, https://doi.org/10.1007/s12161015-0255-y.

[18] G. Gurdeniz, B. Ozen, Detection of adulteration of extra-virgin olive oil by chemometric analysis of mid-infrared spectral data, Food Chem. 116 (2009) 519-525, https://doi.org/10.1016/j.foodchem.2009.02.068.

[19] Y. Tamaki, G. Mazza, Rapid determination of lignin content of straw using Fourier Transform Mid-Infrared Spectroscopy, J. Agric. Food Chem. 59 (2011) 504-512, https://doi.org/10.1021/jf1036678.

[20] B. Ozturk, D. Yucesoy, B. Ozen, Application of mid-infrared spectroscopy for the measurement of several quality parameters of alcoholic beverages, wine and raki, Food Anal. Methods 5 (2012) 1435-1442, https://doi.org/10.1007/s12161-0129397-3.

[21] W. Elfalleh, M. Ying, N. Nasri, H. Sheng-Hua, F. Guasmi, A. Ferchichi, Fatty acids from Tunisian and Chinese pomegranate (Punica granatum L.) seeds, Int. J. Food Sci. Nutr. 62 (2011) 200-206. doi: 10.3109/09637486.2010.526932.

[22] A. Fadavi, M. Barzegar, M.H. Azizi, Determination of fatty acids and total lipid content in oilseed of 25 pomegranates varieties grown in Iran, J. Food Compos. Anal. 19 (2006) 676-680, https://doi.org/10.1016/j.jfca.2004.09.002.

[23] A.G. Mignani, L. Ciaccheri, A.A. Mencaglia, A. Cimato, Optical absorption spectroscopy for quality assessment of extra virgin olive oil, in: D. Boskou (Ed.), Olive Oil-Constituents, Quality, Health Properties and Bioconversions, Intech, Rijeka, Croatia, 2012, pp. 47-62. doi: 10.5772/1378.

[24] N. Siraj, M.A. Shabbir, M.R. Khan, K.U. Rehman, Preventing oxidation of canola and sunflower oils by addition of pomegranate seed oil, Acta Aliment. 48 (2019) 18-27, https://doi.org/10.1556/066.2018.0005.

[25] O. Uncu, B. Ozen, A comparative study of mid-infrared, UV-Visible and fluorescence spectroscopy in combination with chemometrics for the detection of adulteration of fresh olive oils with old olive oils, Food Control 105 (2019) 209-218, https://doi.org/10.1016/j.foodcont.2019.06.013.
[26] A. Hirri, M. Bassbasi, S. Souhassou, F. Kzaiber, A. Oussama, Prediction of polyphenol fraction in virgin olive oil using mid-infrared attenuated total reflectance attenuated total reflectance accessory-mid-infrared coupled with partial least squares regression, Int. J. Food Prop. 19 (2016) 1504-1512, https://doi.org/10 1080/10942912.2015.1059854.

[27] F.N. Arslan, G. Akin, S.N.K. Elmas, I. Yilmaz, H.G. Janssen, A. Kenar, Rapid detection of authenticity and adulteration of cold pressed black cumin seed oil: A comparative study of ATR-FTIR spectroscopy and synchronous fluorescence with multivariate data analysis, Food Control 98 (2019) 323-332, https://doi.org/10. 1016/j.foodcont.2018.11.055.

[28] P. Jiménez-Sotelo, M. Hernández-Martínez, G. Osorio-Revilla, O.G. Meza-Márquez F. García-Ochoa, T. Gallardo-Velázquez, Use of ATR-FTIR spectroscopy coupled with chemometrics for the authentication of avocado oil in ternary mixtures with sunflower and soybean oils, Food Addit. Contam., Part A 33 (2016) 1105-1115, https://doi.org/10.1080/19440049.2016.1203073.

[29] G. Ozulku, R.M. Yildirim, O.S. Toker, S. Karasu, M.Z. Durak, Rapid detection of adulteration of cold pressed sesame oil adultered with hazelnut, canola, and sunflower oils using ATR-FTIR spectroscopy combined with chemometric, Food Control 82 (2017) 212-216, https://doi.org/10.1016/j.foodcont.2017.06.034.

[30] H. Ali, M. Saleem, M.R. Anser, S. Khan, R. Ullah, M. Bilal, Validation of fluorescence spectroscopy to detect adulteration of edible oil in extra virgin olive oil (EVOO) by applying chemometrics, Appl. Spectrosc. 72 (2018) 1371-1379, https://doi.org/10 1177/0003702818768485.

[31] N. Dupuy, Y. Le Dréau, D. Ollivier, J. Artaud, C. Pinatel, J. Kister, Origin of French virgin olive oil registered designation of origins predicted by chemometric analysis of synchronous excitation - emission fluorescence spectra, J. Agric. Food Chem. 53 (2005) 9361-9368, https://doi.org/10.1021/jf051716m.

[32] S.N.K. Elmas, F.N. Arslan, G. Akin, A. Kenar, H.G. Janssen, I. Yilmaz, Synchronous fluorescence spectroscopy combined with chemometrics for rapid assessment of cold-pressed grape seed oil adulteration: Qualitative and quantitative study, Talanta 196 (2019) 22-31, https://doi.org/10.1016/j.talanta.2018.12.026. 